\title{
Capacités d'adaptation et développement à effet d'intégration
}

\section{Résultats du programme de travail 2003-2004 du Centre de développement de I'OCDE}

\author{
par Ulrich Hiemenz
}

\section{De l'importance du renforcement}

des capacités

Les processus de Doha et de Monterrey, ainsi que des initiatives régionales comme le NEPAD, témoignent de l'émergence d'un nouveau partenariat mondial entre les pays de I'OCDE et les pays en développement. Ce partenariat se caractérise par une coopération plus étroite basée sur des stratégies de développement national, avec un effort accentué des pays de I'OCDE en vue de faciliter l'atteinte des Objectifs du Millénaire pour le développement et une participation accrue des pays en développement à l'élaboration des règles internationales. Des progrès ont été accomplis sur tous ces fronts, démontrant que le but poursuivi par l'ensemble des parties prenantes est bien de renforcer l'effet d'intégration de la mondialisation - à la fois entre pays et au sein même des pays. Cependant, les nouvelles responsabilités endossées par les pays en développement pour impulser leur propre développement et contribuer à façonner le paysage économique mondial ne se doublent pas de capacités publiques et privées suffisantes, dans les pays pauvres en particulier. Le partenariat mondial que les pays de l'OCDE et les pays en développement sont en train d'échafauder devra donc avant tout aider ces pays à surmonter les défis de la mondialisation tout en consolidant leurs capacités d'adaptation.
Chacun sait que le renforcement des capacités résulte directement du développement lui-même. Dès lors que la croissance facilite l'augmentation des rentrées fiscales et des profits, les pouvoirs publics et le secteur privé sont en mesure de consolider les institutions et d'investir dans des infrastructures de meilleure qualité comme dans le capital productif. Pour autant, l'enclenchement du processus de développement présuppose un certain niveau de capacités publiques et privées. Cette question était au cœur du programme de travail 2003/04 du Centre de développement. Les leçons tirées des analyses menées dans le cadre de ce programme sont présentées dans les paragraphes qui suivent. L'annexe reprend les publications qui ont servi de base à cette synthèse.

\section{Que peuvent faire les pays de I'OCDE ?}

A l'ère de la mondialisation de l'économie, les interactions entre pays de I'OCDE et pays en développement sont multiples, qu'il s'agisse des relations macro-économiques, des flux réciproques de ressources (IDE et migrations), des relations commerciales ou des activités de coopération au développement. Dans tous ces domaines, les pays de I'OCDE peuvent soutenir, directement et indirectement, les pays en développement qui cherchent à prendre part à la division internationale du travail et à parvenir à un modèle de développement durable. 
Le Centre a privilégié quatre grands domaines dans lesquels l'action des pays de l'OCDE peut faire une différence : le maintien d'un environnement macroéconomique stable et de taux d'intérêt raisonnables ; le soutien à l'atténuation des risques; le renforcement des opportunités commerciales grâce à une cohérence accrue de leurs politiques; et l'augmentation des flux financiers destinés à l'accomplissement des OMD.

\section{Éviter de nouvelles crises du marché}

Depuis à peine une dizaine d'années, l'interdépendance macro-économique entre pays de l'OCDE et pays en développement est moins à sens unique qu'auparavant, grâce surtout au rattrapage des pays asiatiques et notamment de la Chine et de I'Inde. Le modèle de croissance de ces pays pourrait avoir un réel impact sur l'activité mondiale. Parallèlement, la constitution de réserves de change dans les pays asiatiques après la crise financière qui les a frappés pourrait influencer la volatilité des taux d'intérêt et de change. Cela étant, les cycles conjoncturels dans les pays de I'OCDE ainsi que leurs politiques de change et de taux d'intérêt pèsent encore fortement sur la fixation des taux d'intérêt réels internationaux comme sur l'accès aux flux financiers et leur versatilité. A cet égard, les lourds déficits budgétaires américains, qui vont de pair avec une politique monétaire accommodante, pourraient bien composer un cocktail explosif qui n'épargnerait ni les pays de l'OCDE ni les pays en développement.

\section{Atténuer les risques de catastrophes naturelles}

De toute évidence, les pays de l'OCDE n'auraient pas pu faire grand chose pour prévenir les catastrophes naturelles telles que le raz-de-marée en Asie du Sud-Est, les tremblements de terre en Iran (et auparavant en Turquie) ou encore le cyclone Mitch en Amérique centrale. L'analyse de ces événements montre pourtant que l'adoption anticipée de dispositions préventives aurait pu au moins sauver de nombreuses vies humaines dans les zones ravagées. Les systèmes d'alerte, les normes de construction ou la planification de mesures d'urgence sont largement à même de limiter le nombre de victimes humaines des catastrophes naturelles. Les pays de l'OCDE ont un rôle de premier plan à jouer pour l'adoption de telles dispositions préventives, en fournissant les technologies ad hoc et en aidant les pays en développement à financer les investissements nécessaires. De même et au-delà de l'aide d'urgence qu'ils apportent, les pays de l'OCDE peuvent participer à la phase de reconstruction et assumer là un rôle encore plus utile. Les populations les plus pauvres - qui n'ont les moyens ni d'épargner ni de souscrire une assurance adaptée - sont souvent aussi les plus lourdement frappées par les catastrophes naturelles. C'est pourquoi les pays de l'OCDE ne devraient pas se contenter d'allouer des fonds au titre de I'APD pour aider ces populations pendant la phase de reconstruction; ils devraient aussi coopérer à des projets impliquant le secteur privé et les marchés financiers internationaux, afin de permettre aux personnes démunies de s'assurer à un prix abordable contre les chocs liés aux catastrophes naturelles.

\section{Développer les opportunités commerciales}

Pour pouvoir rattraper les pays avancés, les pays en développement devront s'intégrer davantage dans l'économie mondiale et consolider leurs capacités commerciales. Cette intégration accrue passe notamment par l'accès aux marchés des pays de l'OCDE - ce qui a été officiellement reconnu à l'occasion des négociations de Doha. L'enjeu du commerce ne se résume cependant pas au problème des barrières tarifaires et non tarifaires. A l'ère de la mondialisation, les mouvements des facteurs font partie intégrante des relations économiques internationales. Les politiques des pays de l'OCDE qui encouragent par exemple les IDE dans les pays en développement peuvent contribuer à renforcer les capacités commerciales de ces derniers et accentuer les bienfaits de la libéralisation des échanges. Il faut noter ici que les effets bénéfiques des différentes politiques des pays de l'OCDE sur les pays en développement peuvent se renforcer mutuellement. Le Centre a mis en évidence cette synergie des politiques à propos de l'expérience des pays d'Asie de l'Est.

Que les pays de cette région aient pu se développer rapidement tient à la fois à l'impact significatif et largement positif des politiques des pays de l'OCDE en termes $d^{\prime}$ incitations à la croissance, et au fait que ces pays ont mis en place les cadres politiques requis et mobilisé leurs capacités de réponse, humaines et institutionnelles. L'action des pays de l'OCDE en faveur de la cohérence des politiques de développement s'est déclinée en quatre directions :

- contribuer à maintenir une stabilité politique et macroéconomique propice à une croissance tirée par le secteur privé ;

- promouvoir un marché international des biens et des services ouvert et prévisible, fonctionnant sur une base multilatérale et non discriminatoire, ainsi qu'une régulation rationnelle des flux migratoires et un soutien aux échanges ;

- renforcer les structures de gouvernance régissant les investissements et les capitaux internationaux, afin de favoriser l'entrée de capitaux et de technologies dans les pays en développement et de contribuer à la stabilité financière ;

- accroître l'efficacité de l'aide, bilatérale et multilatérale, grâce à une harmonisation et un alignement, en privilégiant la croissance et le renforcement des capacités humaines et institutionnelles. 


\section{Augmenter les flux financiers pour atteindre les OMD}

De l'avis général, les flux financiers actuels des pays de I'OCDE vers les pays en développement ne suffiront pas pour accomplir les OMD d'ici 2015. Les décideurs des pays de I'OCDE ont donc réagi à ce constat en réfléchissant à deux grandes options : accroître le volume et l'efficacité de l'aide ; et proposer des solutions alternatives de financement des OMD. Dans ces deux domaines, le Centre a apporté sa contribution aux débats. Une réallocation des flux d'APD en direction de la production de biens publics pourrait en renforcer l'efficacité vis-à-vis des OMD sans compromettre pour autant les modes traditionnels de coopération au développement avec les pays en pauvres. Outre l'APD, il existe des solutions complémentaires sans effet de distorsion pour augmenter les flux financiers, notamment dans le domaine de l'ingénierie financière - à l'instar de la facilité de financement international (IFF) ou des garanties publiques. Ces options paraissent très prometteuses dans la mesure où elles pourraient développer un sentiment d'appropriation dans les pays bénéficiaires et permettraient d'allouer les ressources, limitées, à des projets présentant un fort rendement potentiel. Cela étant, les discussions actuelles entre une petite dizaine de pays laissent à penser que l'on est encore loin d'un consensus sur ces questions.

Le manque de sensibilisation de I'opinion publique aux enjeux des OMD est l'un des obstacles à la formulation de solutions politiquement acceptables pour réunir les financements nécessaires. Tant que les Européens ne seront que 12 pour cent à connaître ces objectifs, la société civile n'aura pas les moyens de faire pression sur les hommes politiques pour parvenir à un accord. Le Centre collabore donc avec les responsables de l'information des pays membres du CAD pour collecter des données pertinentes et coordonner des campagnes de sensibilisation en faveur d'un financement accru des OMD.

Quand les pays en développement doivent faire preuve d'initiative

$S^{\prime}$ il est un défi majeur pour les institutions et les politiques des pays en développement, c'est bien celui des capacités - pour être en mesure de saisir les nouvelles opportunités et d'enclencher un processus de développement largement partagé. C'est à ces pays de concevoir des stratégies adéquates pour accroître leur compétitivité nationale, mobiliser le secteur privé, édifier des institutions chargées d'orienter et de consolider les transactions commerciales, améliorer les infrastructures et lutter contre la pauvreté. Les pays de I'OCDE peuvent appuyer les efforts de ces pays en transférant leur savoir-faire et en assurant une aide financière - mais l'initiative doit venir des pays en développement partenaires, pour favoriser l'apparition d'un sentiment d'appropriation et de responsabilité. Les travaux du Centre sur le coût du capital dans les pays en développement, les obstacles à la constitution de capacités concurrentielles et les conditions institutionnelles préalables à la réduction de la pauvreté s'inscrivent dans ce contexte.

\section{Promouvoir les investissements}

La baisse des taux d'intérêt intérieurs et, par conséquent, du loyer de l'argent, est essentielle à la promotion de l'investissement et de la croissance économique. La zone rand (Afrique du Sud, Lesotho, Namibie et Swaziland) illustre bien l'intérêt de concevoir des politiques de réduction du coût du capital dans toute l'Afrique australe, dans la mesure où les taux d'intérêt des devises locales sont déterminés par ceux des transactions libellées en rand. La réduction du niveau et de la volatilité de la prime sur le rand a donc un impact sur les taux d'intérêt de tous les pays de la région. L'abaissement et la stabilisation de cette prime nécessiteront d'accroître la liquidité du marché financier, de faciliter l'accès des entreprises africaines aux marchés financiers d'Afrique du Sud, d'encourager l'épargne intérieure et de valoriser l'image internationale du rand. Une fois ces politiques en place, Johannesburg pourrait devenir un pôle financier régional canalisant des ressources bon marché vers ses voisins.

\section{Renforcer les atouts concurrentiels}

Les pays en développement ont crucialement besoin d'augmenter la productivité de leurs industries manufacturières, c'est l'un des piliers de l'élaboration des politiques. L'amélioration des infrastructures, du capital physique, de l'éducation et de l'efficacité globale entraîne des gains de productivité. Pris ensemble et conjugués, ces facteurs indiquent la place réelle d'un pays au « palmarès » de la productivité. Plus qu'une simple comparaison, ces classements permettent d'identifier les facteurs clés propres à chaque pays ou à chaque région et nécessitant une intervention prioritaire. L'analyse montre qu'en Afrique subsaharienne, les principaux obstacles à la compétitivité sont le manque d'infrastructures, notamment pour la fourniture d'énergie, et le faible niveau d'éducation. Ce qui n'autorise cependant pas à négliger totalement les autres domaines... L'une des règles de l'élaboration de politiques impose de s'occuper de tous ces facteurs pour éviter toute influence négative des uns sur les autres venant obérer les gains de productivité indispensables à la lutte contre la pauvreté et à la concrétisation de certains des OMD.

Pour de nombreux pays pauvres, en Afrique et ailleurs, la dépendance à l'égard des matières premières est vécue comme un obstacle à la croissance des industries 
manufacturières et à la diversification des exportations. Le Centre a pourtant démontré qu'il n'y a pas forcément de « malédiction » des ressources naturelles - celles-ci ne condamnent pas les pays à faible revenu au sousdéveloppement, bien au contraire ; elles peuvent servir de tremplin pour une croissance durable tirée par les exportations. Potentiellement, les secteurs dépendant de ressources naturelles peuvent favoriser la diversification des exportations. Les données « miroir » de l'OCDE sur les échanges reflètent une diversité des voies vers la diversification - incluant les industries exploitant les ressources naturelles et de transformation des produits primaires. Rares sont pourtant les pays à faible revenu à exploiter de telles opportunités. Cela tient à la lenteur intrinsèque du processus de diversification des exportations, qui doit pouvoir s'appuyer sur une stratégie adéquate et cohérente combinant vision, coordination et gestion d'intérêts conflictuels. En outre, l'analyse des services d'appui au commerce menée dans deux pays africains met en évidence un décalage entre les besoins du secteur privé et les services disponibles, ainsi qu'un piètre développement institutionnel des réseaux de soutien au commerce et aux investissements. Importantes pour I'Afrique, ces leçons relatives au renforcement des capacités commerciales le sont également pour d'autres pays à faible revenu en Amérique latine et en Asie.

Enfin, les systèmes de gouvernance sont - pour diverses raisons que nous allons évoquer - un déterminant majeur du volume et de l'allocation des investissements dans les économies en développement et émergentes. Un examen attentif des institutions chargées de la gouvernance est donc un aspect essentiel du développement et du renforcement de la compétitivité internationale d'un pays. Parfaitement conscients de cette corrélation, les pays africains ont introduit - sous l'égide du NEPAD - le mécanisme africain d'évaluation par les pairs. C'est dans cette optique que s'inscrivent également les Perspectives économiques en Afrique, fruit d'une collaboration entre la Banque africaine de développement et le Centre. Cet ouvrage propose une analyse comparée des dynamiques internes et externes du développement de tous les grands pays africains. Les institutions et les décideurs africains peuvent donc s'y référer pour jauger l'efficacité des politiques adoptées dans certains pays ou plaider en faveur de réformes des politiques. Pour être crédible, un tel instrument analytique se doit d'être intégré par les institutions africaines - les pays de l'OCDE pouvant contribuer à développer les capacités requises.

\section{Consolider et adapter les institutions}

Il est désormais largement acquis que les institutions jouent un rôle de premier plan dans la promotion d'un développement économique favorable aux pauvres. Les institutions officielles et les systèmes de gouvernance encadrent l'économie de marché et la fourniture de biens publics. Dans le cas des pays en développement et dans la perspective de parvenir à un développement durable, il convient cependant d'intégrer une autre catégorie d'institutions, plus informelles : il s'agit notamment des structures familiales et de parenté ou des traditions, ainsi que des règles civiques et sociales que l'on évoque communément sous le terme de « culture ». Alors que la concurrence s'intensifie et que les échanges interculturels s'accélèrent, ces institutions sociales traditionnelles peuvent favoriser - ou au contraire contrarier - l'émergence de nouvelles opportunités économiques et sociales.

La culture détermine notamment la place des femmes dans la société. Le Centre a mis en évidence de profondes disparités entre les régions. D'une manière générale, la discrimination fondée sur le sexe est moins marquée en Amérique latine et en Asie de l'Est qu'en Afrique subsaharienne, en Asie du Sud et dans les pays d'Afrique du Nord et du Moyen-Orient (MENA). Ensuite, et ce point est plus important, la religion n'est pas la cause principale de l'inégalité entre les sexes - car au sein même des grandes religions, le rôle des femmes s'apprécie avec une certaine latitude. Enfin, l'existence d'un cadre institutionnel biaisé au détriment des femmes compromet le développement, ce qui n'a rien d'étonnant dans la mesure où il concerne les capacités de la moitié de la population à participer aux activités économiques.

L'adaptation des institutions sociales aux défis du développement économique exige une détermination de la part des pouvoirs publics à remettre en cause des traditions et des privilèges vieux de plusieurs siècles. La situation des femmes n'a guère de chances de s'améliorer sensiblement tant que l'on continuera de ne s'intéresser qu'à leur éducation, à leur prise en charge médicale et à leur accès au crédit. Pour fondamentales qu'elles soient, ces mesures doivent aller de pair avec une évolution des comportements et pratiques discriminatoires tant au niveau de l'État que dans les communautés. Lorsque la coutume interdit aux filles de sortir de chez elles, on ne voit pas ce qu'une école de filles changera à la donne.

Les travaux sur la décentralisation de l'élaboration des politiques aux régions ont mis en évidence les interconnexions entre institutions formelles et informelles ainsi que leur influence sur l'impact des réformes. La communauté internationale s'intéresse de plus en plus à la décentralisation en tant qu'instrument potentiel de la lutte contre la pauvreté. Pourtant, les travaux du Centre n'ont pas identifié de relation évidente entre décentralisation et réduction de la pauvreté. Bien au contraire, dans certains des pays les plus pauvres dotés $d$ 'institutions peu efficaces et en proie à des conflits politiques, la décentralisation pourrait même aggraver les choses. Deux grandes leçons découlent de ce constat : tout d'abord, les bailleurs de fonds devraient se garder d'accorder la priorité à la décentralisation dans les pays 
où le pouvoir central remplit à peine sa mission de base et ne voit pas l'intérêt d'accorder davantage de pouvoir et de ressources aux autorités locales - car elle pourrait avoir un effet inverse sur l'objectif de réduction de la pauvreté. En revanche, dans les pays qui répondent aux conditions préalables minimales, la décentralisation peut fortement contribuer à la lutte contre la pauvreté. La décentralisation en faveur des pauvres passe par une compréhension précise des facteurs clés influençant le processus lui-même - à savoir les institutions informelles qui régissent les comportements aux échelons territoriaux.

Les institutions formelles et informelles ne sont pas seules à entretenir des relations croisées: les institutions formelles interagissent aussi entre elles. Il en va ainsi de l'interdépendance mutuelle entre la gouvernance publique et la gouvernance d'entreprise, essentielle. La gouvernance d'entreprise peut jouer un rôle majeur dans les pays en développement et en transition, ainsi que dans les économies de marché émergentes. Dans les pays pauvres comme dans les pays à revenu intermédiaire, dans les pays où seules les actions d'un petit nombre d'entreprises sont largement négociées comme dans ceux où les marchés boursiers sont plus actifs, la gouvernance d'entreprise permet d'accroître, en en réduisant le coût, les flux financiers dont les entreprises ont besoin pour financer leurs investissements dans des actifs réels, pour accroître la liquidité dans l'économie du pays considérée globalement, et pour obtenir des gains de productivité à long terme. Les forces qui œuvrent en faveur d'une amélioration de la gouvernance d'entreprise se trouvent aussi bien dans les entreprises dont les besoins de financement augmentent que chez les grands investisseurs institutionnels (notamment les fonds de pensions et autres investisseurs à long terme basés dans les pays de I'OCDE) et, de plus en plus, dans les pays en développement ou en transition et les économies de marché émergentes. Et les forces qui opposent une forte résistance à l'amélioration de la gouvernance d'entreprise se retrouvent dans les rangs de nombreux actionnaires majoritaires et autres détenteurs de postes clés (insiders) au sein des entreprises, privées ou publiques, qui constituent souvent des coalitions distributives solidement enracinées. La situation est particulièrement délicate lorsque les participations croisées, les catégories d'actions multiples et, en particulier, des structures d'actionnariat pyramidales, servent à générer des rentes de contrôle des entreprises.

L'importance des coalitions distributives dans les pays en développement - qui sont autant d'obstacles au développement et à l'amélioration de la gouvernance d'entreprise - ainsi que le risque accru de captation de la réglementation dans les systèmes clientélistes de gouvernance fondés sur les relations individuelles, viennent conforter l'idée qu'une bonne gouvernance d'entreprise exige une bonne gouvernance publique, et réciproquement. Le développement impose de substituer la force du droit à la force des particuliers dans les institutions de gouvernance d'entreprise comme dans celles en charge de la gouvernance publique. L'objectif est d'instaurer une culture de la gouvernance propice à la production et à la confiance, mais aussi à un partage du pouvoir et des informations, profitable à tous. Pour ce faire, il faut instaurer un processus de fixation de règles et de renforcement des institutions qui, surtout dans les pays en développement les plus pauvres, passe probablement par une situation temporaire où les autorités publiques jouent le rôle de point focal du système de gouvernance. 


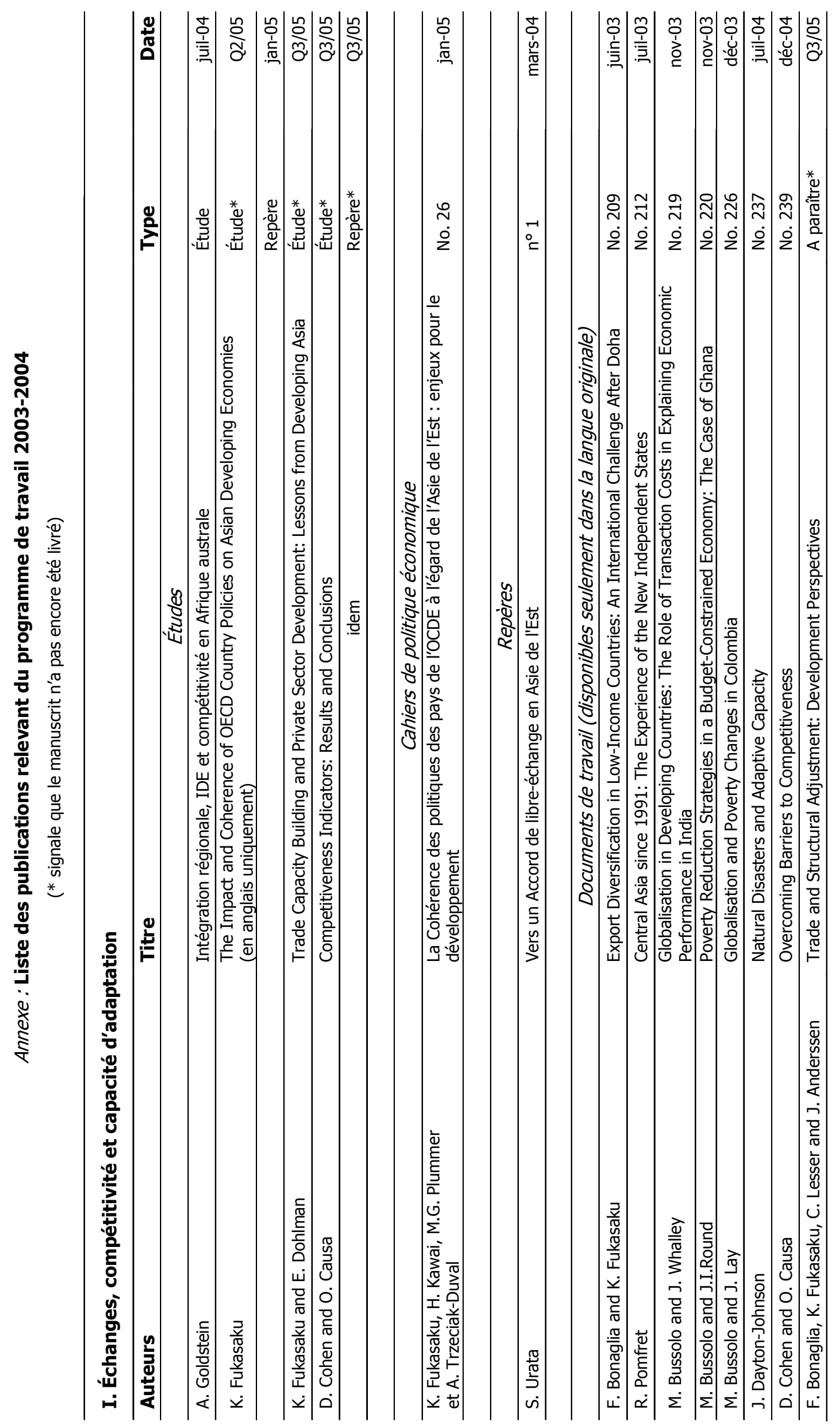




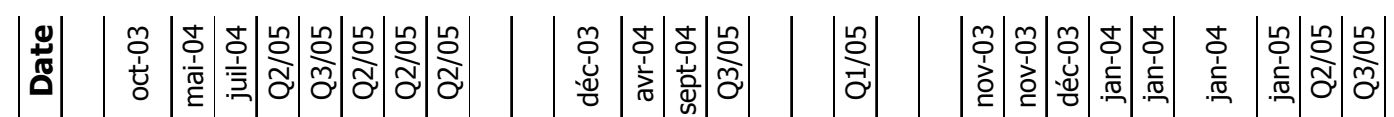

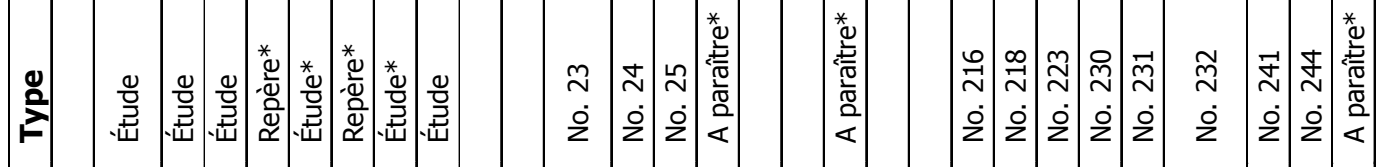

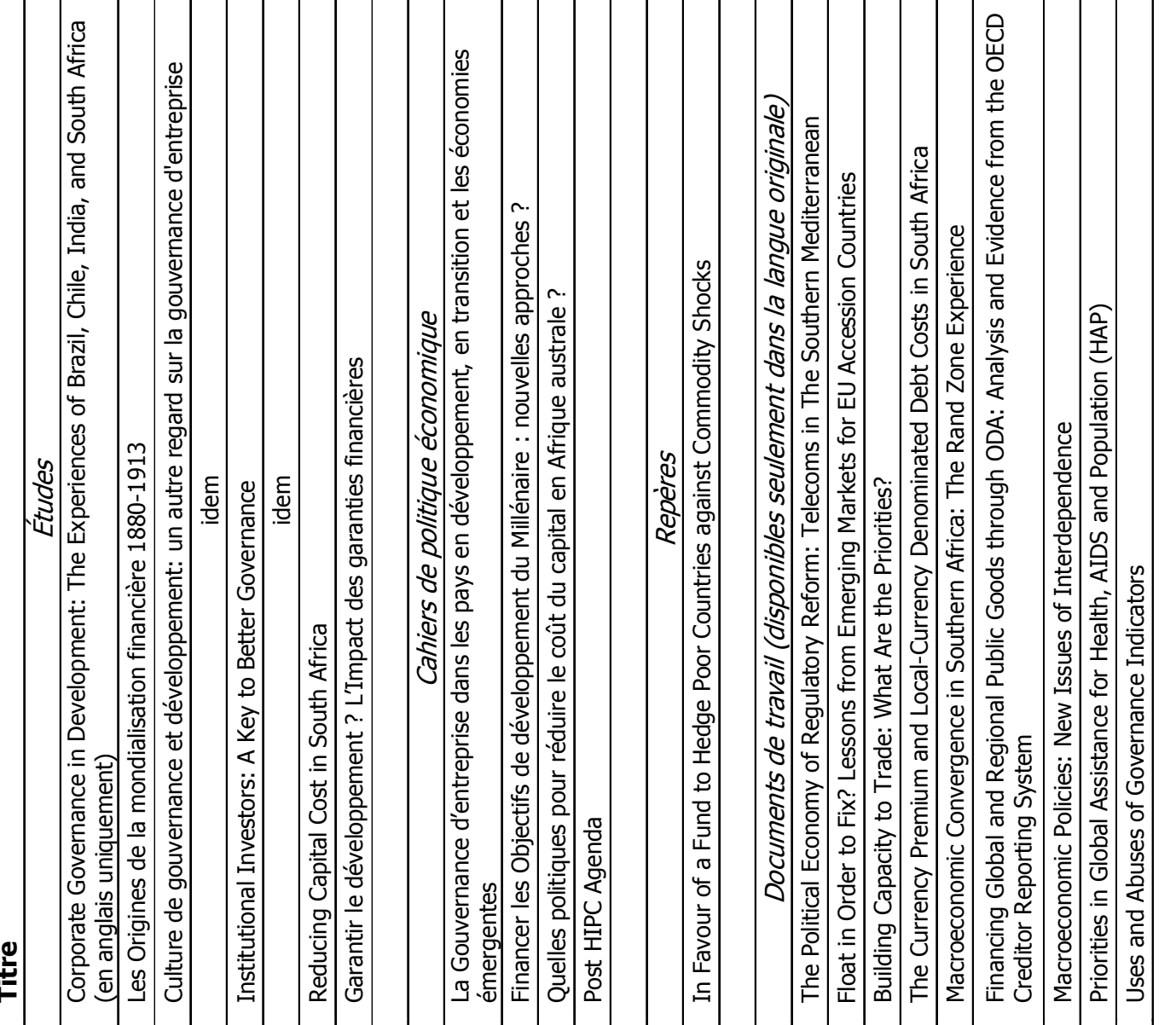

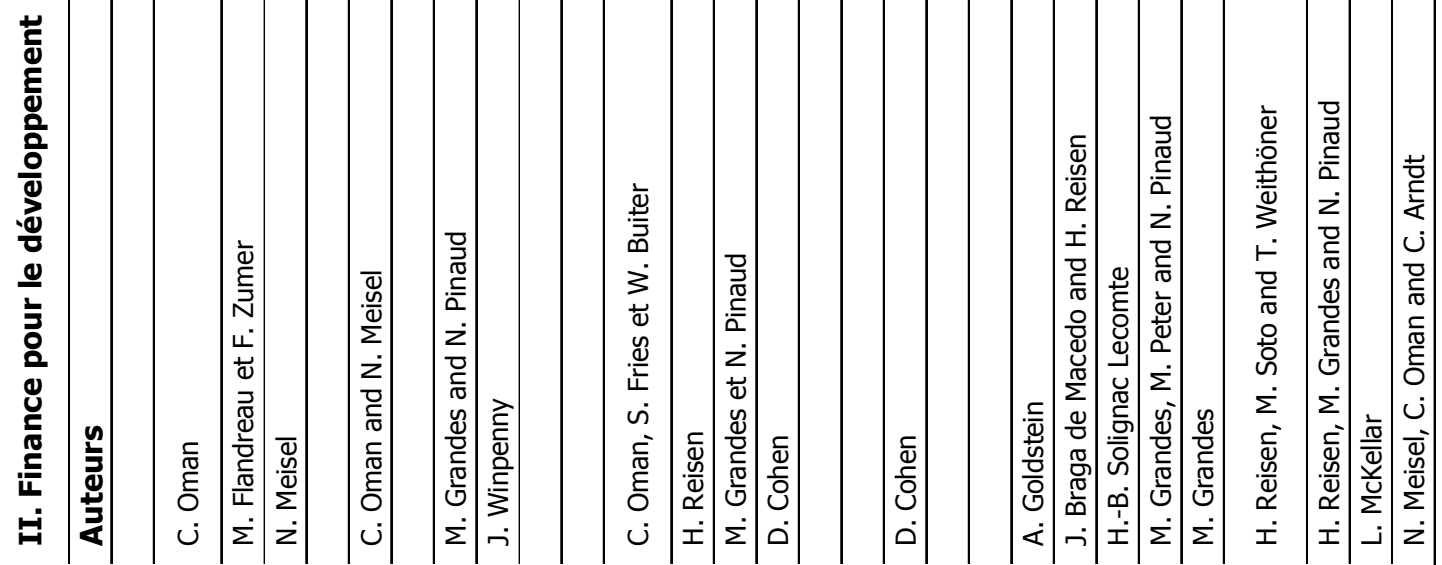




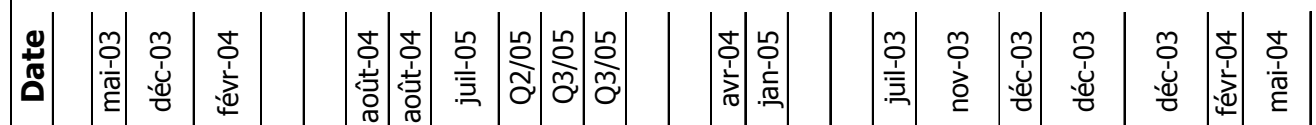

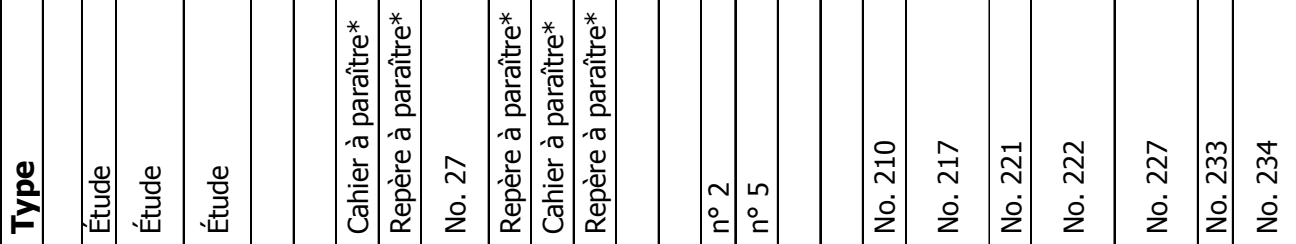

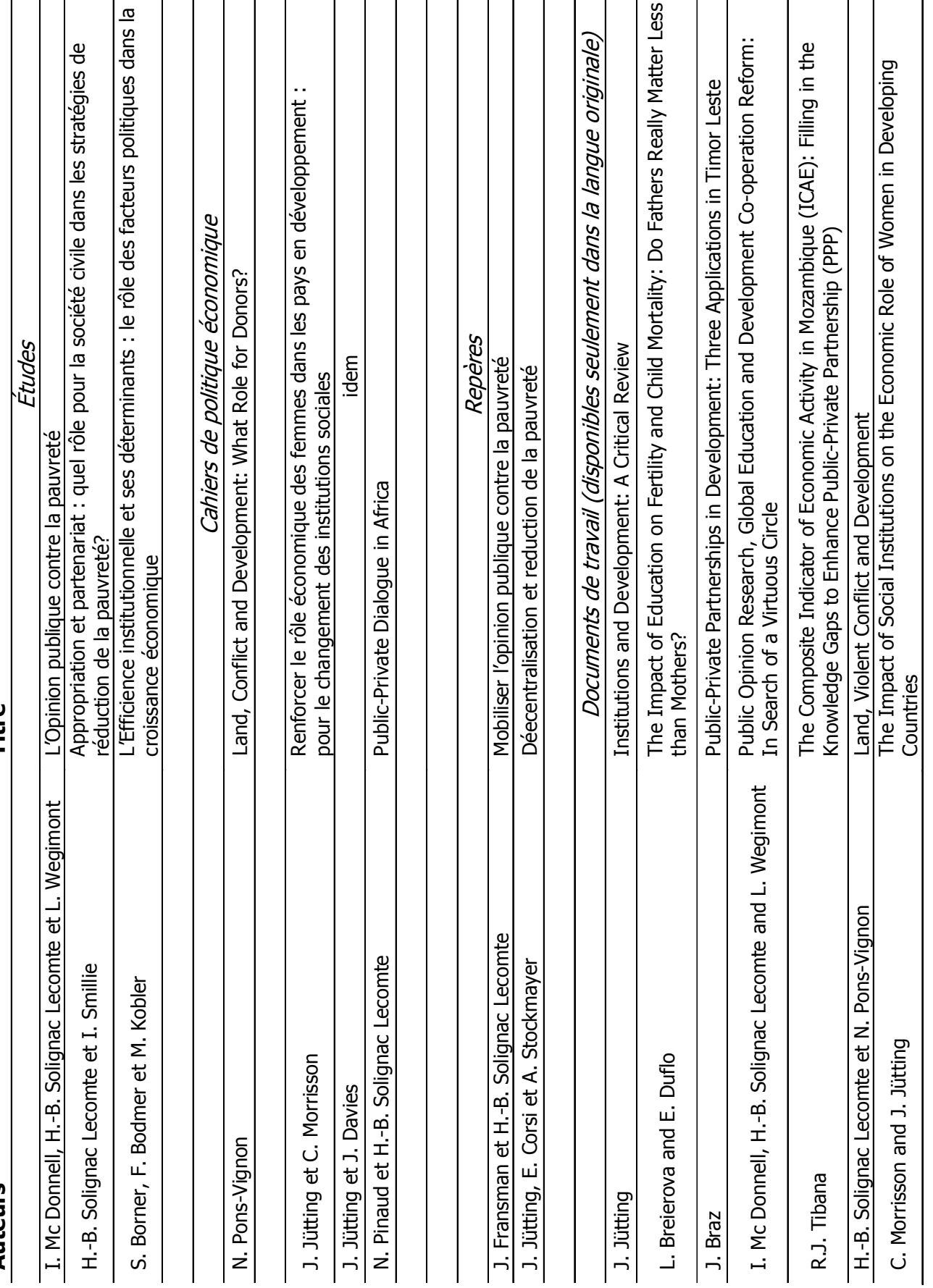




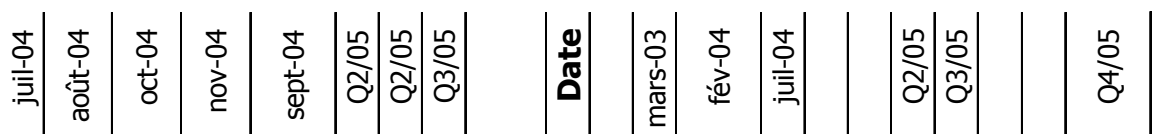

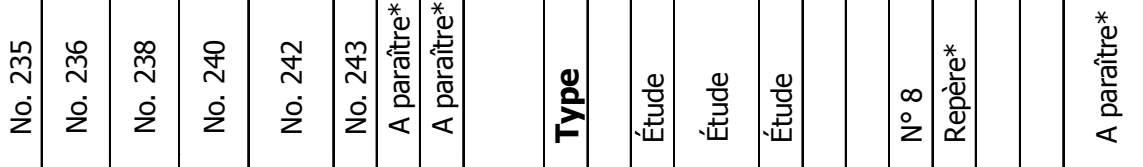
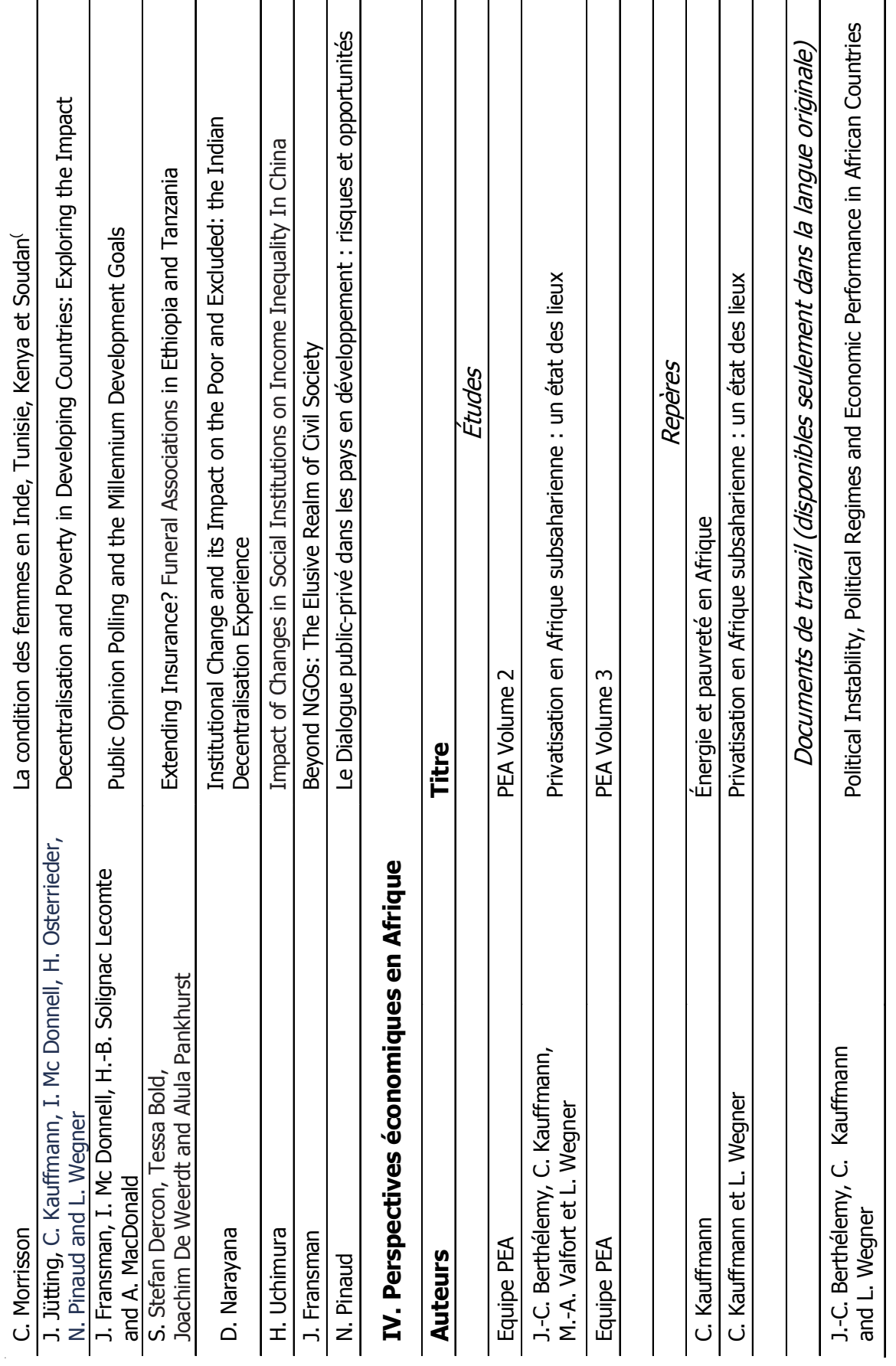
Pour consulter les Repères du Centre de développement

www.oecd.org/dev/reperes

\section{les Cahiers de politique économique}

www.oecd.org/dev/cahiers

ou les nouveaux Documents de travail

www.oecd.org/dev/dt

OCDE $《 O$ 